\title{
A Noise and Mismatches of Delay Cells and Their Effects on DLLs
}

\author{
Mohammad Gholami \\ Dept. of Electrical and Computer Engineering, Babol Noshirvani University of Technology, Babol, Iran \\ E-mail: mgh_elec@yahoo.com \\ Gholamreza Ardeshir \\ Dept. of Electrical and Computer Engineering, Babol Noshirvani University of Technology, Babol, Iran \\ E-mail:g.ardeshir@nit.ac.ir \\ Hossein Miar-Naimi \\ Dept. of Electrical and Computer Engineering, Babol Noshirvani University of Technology, Babol, Iran \\ E-mail:h_miare@nit.ac.ir
}

\begin{abstract}
Jitter is one of the most important parameters in design of delay locked loop (DLL) based frequency synthesizer. In this paper noise and mis matches of conventional delay cells which are mainly used in the DLLs architecture are introduced completely. First, time domain equations related to noise and mis matches of conventional delay cells are reported. Then, these equations are used to calculate jitter of DLL due to mis match and noise of delay cells. At last closed form equations are obtained which can be used in the designing of low jitter DLLs. To validate these equations, a conventional DLL is designed in TSMC 0.18um CMOS Technology.
\end{abstract}

\section{Introduction}

Nowadays, Delay Locked Loops (DLL) and Phase Locked Loops are widely used in high-speed systems, frequency synthesizers [1], RAM [2], clock synchronization and clock and data recovery circuits [3]. They are unavoidable parts in communication systems. As DLLs shows better jitter performance than PLLs, they used more when jitter should be minimized [4]. Conventional DLLs are first order system, hence they are inherently stable. Also, they propose lower jitter and smaller chip area than PLLs [5]. A comparison between PLLs and DLLs are abbreviated in Table 1.

Index Terms - Mis matches, Noise, Phase Errors, Jitter, DLL, Delay Locked Loo

Table 1: Comparison of PLLs and DLLs

\begin{tabular}{|l|l|}
\hline \multicolumn{1}{|c|}{ DLLs } & \multicolumn{1}{c|}{ PLLs } \\
\hline First order system and stable & Higher order systems and can be unst able \\
\hline Fast locking time & Slow locking time \\
\hline Easier to design & Hard to design \\
\hline Occupy smaller area & Occupy large area \\
\hline No jitter accumulation & Jitter accumulation \\
\hline Small jitter and phase noise & More jitter and phase noise \\
\hline Usually consume lower power & Usually consume more power \\
\hline More dependence to referenceclock & Lower dependence to reference clock \\
\hline Cannot generate fractional multiples of reference clock & Can generate fractional multiples of reference clock \\
\hline
\end{tabular}

Fig. 1 shows the generic RF transceivers architecture [6]. In this figure up-conversion and down-conversion are driven by an oscillator $[7,8]$ which is controlled by frequency synthesizer. DLLs and PLLs are used as a frequency synthesizer in transceivers. Jitter, power consumption and phase noise are important parameters and when there is no need to generate fractional multiple of reference frequency, DLL is used instead of PLL because of its better performance. In addition, DLLs are widely used in dig ital commun ication circuits $[9,10,11]$. 


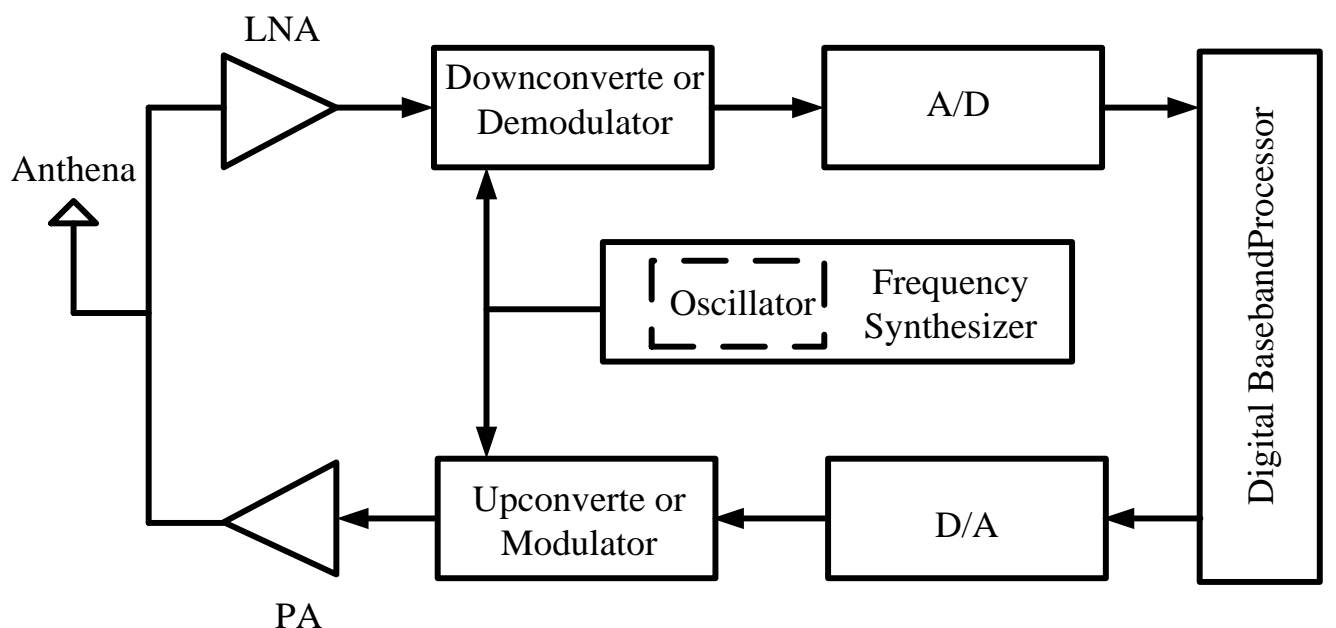

Fig. 1: Generic RF transceiver architecture [6]

In design of frequency multiplier and clock and data recovery circuits, jitter is one of the most important parameters. In [12] analyses and experimental results on the jitter transfer of DLLs are reported. In this work $\mathrm{z}$ domain model of DLL is used to obtain jitter transfer function of whole DLL. In [13] an all-digital is designed to reduce the whole jitter. To achieve this goal a duty cycle correction circuit is used. In [14] design techniques of a multiphase clock generator using a lowjitter delay-locked loop or its array for the developments of high-resolution multi-channel time-to-digital converters is presented. The low-jitter technologies for both a single DLL and an array of DLL are also discussed in [14].

Hence, designing a DLL with lower RMS jitter is of high importance. DLL has four main blocks including Chare Pump (CP), Phase Frequency Detector (PFD), Voltage Controlled Delay Line (VCDL) and Loop Filter. Non-ideal blocks and also mismatches in them result in jitter in the output of DLLs. Hence, developing an equation for describing the jitter performance of DLLs is importance. In this paper, after describing noise and mis match of delay cells, their impacts on DLL's jitter will be analyzed.

In the next section, the conventional DLL-based frequency synthesizer will be explained. Section III describes the jitter due to noisy delay cells. Simu lations and results will be presented in section IV.

\section{Conventional Delay Locked Loop Based Multipliers}

Fig 2 shows the architecture of conventional DLL. As shown in this figure, a conventional DLL consists of a Phase-Frequency Detector (PFD), a Charge Pump (CP), a Loop Filter (LF) and a Voltage Controlled Delay Line (VCDL). In a DLL, phase difference between REF and OUT signals is detected in PFD. The resulted signal at the output of PFD is sent to CP. CP and LF (integrator) generate appropriate value for control voltage of VCDL
(Vcntl) based on phase difference of REF and OUT and accordingly the delay of each delay cell is adjusted. This process is repeated until DLL is locked. It should be mentioned that in lock condition, the input and output of VCDL are in phase and the delay which is produced by VCDL is exactly equal to TREF (TREF is the period of reference clock).This means, in lock condition REF and OUT have exactly one clock period difference.

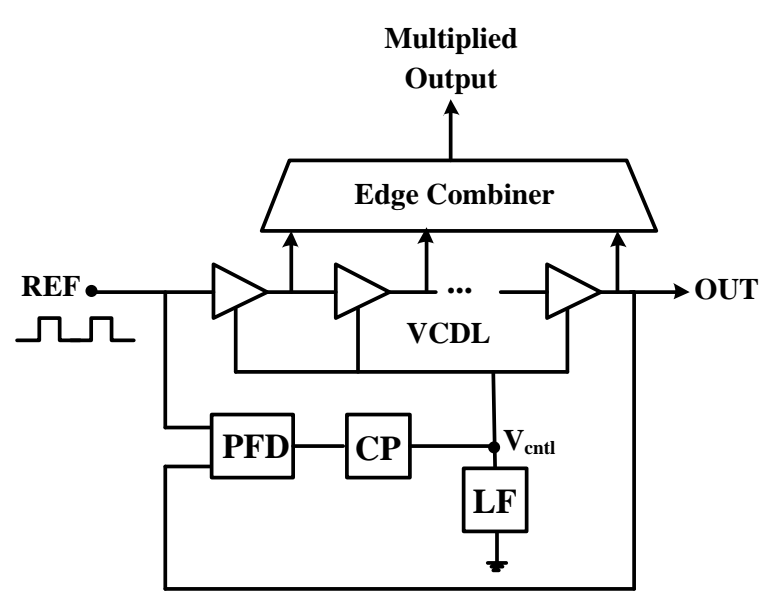

Fig. 2: Conventional DLL-based clock multiplier

An edge combiner is used when multiplied output is needed. In general edge combiner is consist of XOR gates which combine the edges at the outputs of delay cells. Fig. 3 shows the DLLs outputs with five delay stages in the delay chain. As can be seen in this figure five times of reference clock can be generated in this case. In general by $\mathrm{N}$ delay cells in the VCDL, $\mathrm{N}$ times of reference clock can be obtained when $\mathrm{N}$ is odd and $\mathrm{N} / 2$ times of reference clock can be generated when $\mathrm{N}$ is even. In the next section conventional delay cell is introduced and then the noise and mis matches of it will be formulized. At last the effects of noise and mismatches of delay cells will be investigated in DLLs. 


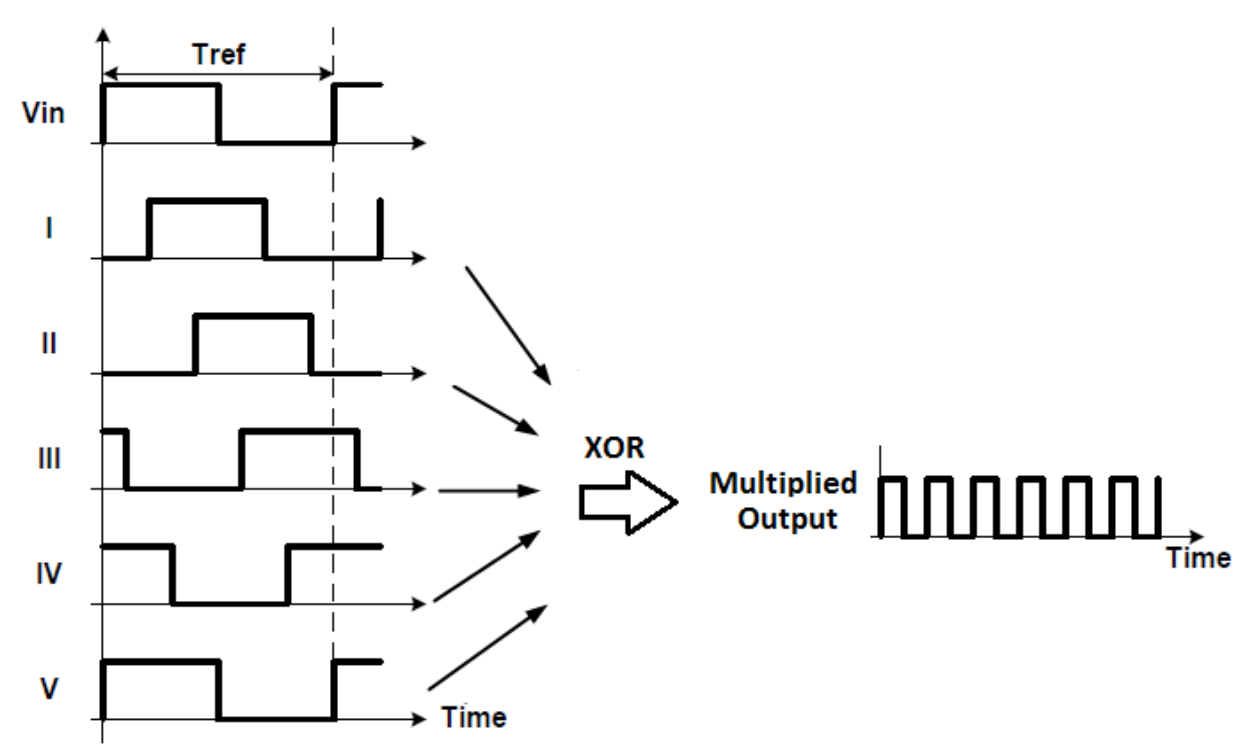

Fig. 3: Waveforms of DLL with five delay cells in the VCDL

\section{Noise and Mis match of Delay Cells and Their Effects on the DLLs}

Jitter in delay cells is caused by noise and mis matching. Here, we analyse jitter produced by noise and mis matching in a conventional delay cell as done in [15].

\subsection{Jitter Due to Noisy Delay Cells}

The delay of a conventional delay cell shown in Fig.4 can be written as:

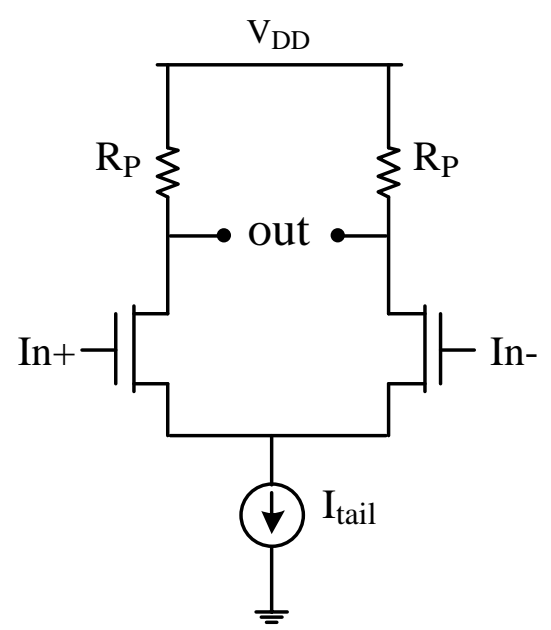

Fig. 4: Conventional Delay cells which is used in DLLs

$$
\tau_{D S}=\frac{V_{S W}}{I_{S S}} \times C_{L} \times \ln 2=R_{P} \times C_{L} \times \ln 2
$$

In this equation $\tau_{D S}$ is the delay of each delay cell, $V_{S W}$ is the output voltage swing, $I_{S S}$ is the tail current of the delay cell, $C_{L}$ is the load capacitor and $R_{P}$ is the equivalent resistance of pMOS load. According to [16], the jitter of delay cells due to noise is:

$$
\sigma\left(\Delta \mathrm{t}_{\mathrm{DS}}\right)=\tau_{\mathrm{DS}} \times \sqrt{\frac{\mathrm{KT}}{\mathrm{C}_{\mathrm{L}}}} \times \frac{\alpha}{\mathrm{V}_{\mathrm{GS}, \mathrm{n}}-\mathrm{V}_{\mathrm{T}, \mathrm{n}}}
$$

Where $\mathrm{K}$ is Boltezman constant, $\mathrm{T}$ is temperature in Kelvin. ${ }^{\alpha}$ is constant parameter based on technology. Also, $\mathrm{V}_{\mathrm{T}, \mathrm{n}}$ is the threshould voltage of nMOS transistor. Calculating variance of both sides of (2):

$$
\sigma^{2}\left(\Delta \mathrm{t}_{\mathrm{DS}}\right)=\tau_{\mathrm{DS}}^{2} \times\left(\frac{\mathrm{KT}}{\mathrm{C}_{\mathrm{L}}}\right) \times\left(\frac{\alpha}{\mathrm{V}_{\mathrm{GS}, \mathrm{n}}-\mathrm{V}_{\mathrm{T}, \mathrm{n}}}\right)^{2}
$$

Hence, by replacing $\tau_{D S}$ from (1) into (3):

$$
\begin{aligned}
\sigma^{2}\left(\Delta \mathrm{t}_{\mathrm{DS}}\right)=\left(\frac{\mathrm{V}_{\mathrm{SW}}}{\mathrm{I}_{\mathrm{SS}}}\right. & \left.\times \mathrm{C}_{\mathrm{L}} \times \ln 2\right)^{2} \\
& \times\left(\frac{\mathrm{KT}}{\mathrm{C}_{\mathrm{L}}}\right) \times\left(\frac{\alpha}{\mathrm{V}_{\mathrm{GS}, \mathrm{n}}-\mathrm{V}_{\mathrm{T}, \mathrm{n}}}\right)^{2}
\end{aligned}
$$

and by simplifying this equation, $\sigma^{2}\left(\Delta t_{D S}\right)$ can be written as:

$$
\sigma^{2}\left(\Delta \mathrm{t}_{\mathrm{DS}}\right)=\frac{(\mathrm{KT}) \times \mathrm{C}_{\mathrm{L}} \times\left(\mathrm{V}_{\mathrm{SW}} \times \ln 2 \times \alpha\right)^{2}}{\mathrm{I}_{\mathrm{SS}}{ }^{2} \times\left(\mathrm{V}_{\mathrm{GS}, \mathrm{n}}-\mathrm{V}_{\mathrm{T}, \mathrm{n}}\right)^{2}}
$$

For DLL with $\mathrm{N}$ delay stages, the jitter at the output of X-th delay stage can be obtained from:

$$
\sigma^{2}\left(\Delta \mathrm{t}_{\mathrm{X}}\right) \approx \mathrm{N} \times \frac{(\mathrm{KT}) \times \mathrm{C}_{\mathrm{L}} \times\left(\mathrm{V}_{\mathrm{SW}} \times \ln 2 \times \alpha\right)^{2}}{\mathrm{I}_{\mathrm{SS}}{ }^{2} \times\left(\mathrm{V}_{\mathrm{GS}, \mathrm{n}}-\mathrm{V}_{\mathrm{T}, \mathrm{n}}\right)^{2}}
$$




\subsection{Jitter of Delay Cells Due to Delay Cells Mismatch}

To find the jitter produced by mismatching in delay cells, we assume that the delay of each delay cell is equal to:

$$
\tau_{\mathrm{DS}_{\mathrm{i}}}=\tau_{\mathrm{DS}_{\text {nom }}}+\tau_{\mathrm{DS}_{\mathrm{error}, \mathrm{i}}}
$$

where $\tau_{\mathrm{DS}_{\mathrm{i}}}$ is the delay of i-th delay cell, $\tau_{\mathrm{DS}_{\text {nom }}}$ is the nominal delay of each delay cell and $\tau_{\mathrm{DS}_{\text {errori }}}$ is the delay error of i-th cell. In lock condition of DLL, the phase difference of input and output of DLL (input and output of VCDL) exactly equal to one period of reference clock. Therefore, Eq. (8) can be written in lock condition for delay of VCDL with $\mathrm{N}$ delay stages as:

$$
\tau_{\mathrm{DS}_{1}}+\tau_{\mathrm{DS}_{1}}+\ldots+\tau_{\mathrm{DS}_{\mathrm{N}}}=\mathrm{T}_{\mathrm{REF}}
$$

By applying (7) to (8):

$$
\begin{gathered}
\tau_{\mathrm{DS}_{\text {nom }}}+\tau_{\mathrm{DS}_{\text {error }, 1}}+\tau_{\mathrm{DS}_{\mathrm{nom}}}+\tau_{\mathrm{DS}_{\text {error }, 2}} \\
+\ldots+\tau_{\mathrm{DS}_{\mathrm{nom}}}+\tau_{\mathrm{DS}_{\text {error }, \mathrm{N}}}=\mathrm{T}_{\mathrm{REF}}
\end{gathered}
$$

This results in:

$$
\tau_{\mathrm{DS}_{\text {nom }}}=\frac{\mathrm{T}_{\mathrm{REF}}-\sum_{i=1}^{\mathrm{N}} \tau_{\mathrm{DS}_{\text {eror }, \mathrm{i}}}}{\mathrm{N}}
$$

and by substituting (10) into (7) we have:

$$
\tau_{\mathrm{DS}_{\mathrm{i}}}=\frac{\mathrm{T}_{\mathrm{REF}}-\sum_{i=1}^{\mathrm{N}} \tau_{\mathrm{DS}_{\text {error }, \mathrm{i}}}}{\mathrm{N}}+\tau_{\mathrm{DS}_{\text {error }, \mathrm{i}}}
$$

In lock condition when there is not jitter, the delay of each cell is equal to $\frac{T_{R E F}}{N}$, and the systematic jitter of $X$ th delay stage can be written as:

$$
\Delta t_{X}=\sum_{j=1}^{X} \tau_{D S_{j}}-\frac{X \times T_{R E F}}{N}
$$

By replacing $\tau_{\mathrm{DS}_{\mathrm{j}}}$ from (11):

$$
\begin{array}{r}
\Delta \mathrm{t}_{\mathrm{X}}=\sum_{j=1}^{\mathrm{X}}\left[\frac{\mathrm{T}_{\mathrm{REF}}-\sum_{j=1}^{N} \tau_{\mathrm{DS}_{\text {error }, \mathrm{j}}}}{\mathrm{N}}+\tau_{\mathrm{DS}_{\text {error }, \mathrm{j}}}\right] \\
-\left(\frac{\mathrm{X} \times \mathrm{T}_{\mathrm{REF}}}{\mathrm{N}}\right)
\end{array}
$$

By expanding this equation:

$$
\begin{aligned}
\Delta \mathrm{t}_{\mathrm{X}}=\left(\frac{\mathrm{X} \times \mathrm{T}_{\mathrm{REF}}}{\mathrm{N}}\right) & +\left(\frac{\mathrm{X}}{\mathrm{N}}\right) \sum_{j=1}^{\mathrm{N}} \tau_{\mathrm{DS}_{\text {error }, \mathrm{i}}} \\
& -\sum_{j=1}^{\mathrm{X}} \tau_{\mathrm{DS}_{\text {error }, \mathrm{j}}}-\left(\frac{\mathrm{X} \times \mathrm{T}_{\mathrm{REF}}}{\mathrm{N}}\right)
\end{aligned}
$$

after simplifying, $\Delta \mathrm{t}_{\mathrm{x}}$ can be written as:

$$
\Delta \mathrm{t}_{\mathrm{X}}=\left(\frac{\mathrm{X}}{\mathrm{N}}\right) \sum_{j=1}^{\mathrm{N}} \tau_{\mathrm{DS}_{\text {error }, \mathrm{i}}}-\sum_{j=1}^{\mathrm{X}} \tau_{\mathrm{DS}_{\text {error } \mathrm{j}}}
$$

By calculating the expected value of (15) after squaring, we reach to:

$$
\begin{aligned}
& \mathrm{E}\left(\Delta \mathrm{t}_{\mathrm{X}}^{2}\right)=\left(\frac{\mathrm{X}}{\mathrm{N}}\right)^{2} \times \mathrm{E}\left(\sum_{i=1}^{\mathrm{N}} \tau_{\mathrm{DS}_{\text {error }, \mathrm{i}}}^{2}\right) \\
& +\mathrm{E}\left(\sum_{j=1}^{\mathrm{X}} \tau_{\mathrm{DS}_{\text {error }, \mathrm{j}}}^{2}\right) \\
& -2\left(\frac{\mathrm{X}}{\mathrm{N}}\right) \times \mathrm{E}\left(\sum_{i=1}^{\mathrm{N}} \tau_{\mathrm{DS}_{\text {error }, \mathrm{i}}} \times \sum_{j=1}^{\mathrm{X}} \tau_{\mathrm{DS}_{\text {error }, \mathrm{j}}}\right)
\end{aligned}
$$

The last portion of (16) can be abbreviated as:

$$
\begin{aligned}
& \mathrm{E}\left(\sum_{i=1}^{\mathrm{N}} \tau_{\mathrm{DS}_{\text {eror }, \mathrm{i}}} \times \sum_{j=1}^{\mathrm{X}} \tau_{\mathrm{DS}_{\text {error }, \mathrm{j}}}\right) \\
& =\mathrm{E}\left(\left[\sum_{i=1}^{\mathrm{X}} \tau_{\mathrm{DS}_{\text {eror }, \mathrm{i}}}+\sum_{i=X+1}^{\mathrm{N}} \tau_{\mathrm{DS}_{\text {erro }, \mathrm{i}}}\right] \times \sum_{j=1}^{\mathrm{X}} \tau_{\mathrm{DS}_{\text {error }, \mathrm{j}}}\right) \\
& =\mathrm{X} \times \mathrm{E}\left(\tau_{\mathrm{DS}_{\text {error }}}^{2}\right)
\end{aligned}
$$

and by replacing it into (16):

$$
\begin{aligned}
& \mathrm{E}\left(\Delta \mathrm{t}_{\mathrm{X}}^{2}\right) \\
& =\left[\left(\frac{\mathrm{X}}{\mathrm{N}}\right)^{2} \times \mathrm{N}+\mathrm{X}-2\left(\frac{\mathrm{X}}{\mathrm{N}}\right) \times \mathrm{X}\right] \times \mathrm{E}\left(\tau_{\mathrm{DS}_{\text {error }}}^{2}\right) \\
& =\left[-\frac{\mathrm{X}^{2}}{\mathrm{~N}}+\mathrm{X}\right] \times \mathrm{E}\left(\tau_{\mathrm{DS}_{\text {error }}}^{2}\right)
\end{aligned}
$$

Or equivalently:

$$
\sigma^{2}\left(\Delta t_{X}\right)=\left[-\frac{\mathrm{X}^{2}}{\mathrm{~N}}+\mathrm{X}\right] \times \sigma^{2}\left(\tau_{\mathrm{DS}_{\text {eror }}}\right)
$$

Equation (19) shows that when $X=0$ or $X=N$, the jitter due to mis matching of delay cells in output of $X$-th delay cells is equal to zero. Also, this equation shows that the maximum jitter in VCDL due to mis matching of delay cells happens in $X=N / 2$. These results can be summarized as: 


$$
\begin{aligned}
& \min \left(\sigma^{2}\left(\Delta \mathrm{t}_{\mathrm{X}}\right)\right) \Rightarrow\left\{\begin{array}{l}
\mathrm{X}=0 \Rightarrow \sigma^{2}\left(\Delta \mathrm{t}_{\mathrm{X}}\right)=0 \\
\mathrm{X}=\mathrm{N} \Rightarrow \sigma^{2}\left(\Delta \mathrm{t}_{\mathrm{X}}\right)=0
\end{array}\right. \\
& \max \left(\sigma^{2}\left(\Delta \mathrm{t}_{\mathrm{X}}\right)\right) \Rightarrow \mathrm{X}=\frac{\mathrm{N}}{2} \Rightarrow \sigma^{2}\left(\Delta \mathrm{t}_{\mathrm{X}}\right)=\frac{3}{4} \mathrm{~N} \times \sigma^{2}\left(\tau_{\mathrm{DS}_{\text {eror }}}\right)
\end{aligned}
$$

\section{Simulation and Results}

A conventional DLL-based frequency multiplier has been designed in TSMC $0.18 \mu \mathrm{m}$ CMOS Technology by ADS simulator to evaluate equations obtained in section III.

It should be mentioned that all building blocks have been designed by CML logic and in differential fashion to have a higher speed DLL. All building blocks of the proposed DLL have been reported in [16].

Circuit implementation of delay cell is shown Fig. 4. In addition PFD and CP which are used in this design are shown in Fig.5 and Fig.6 respectively. This DLL has been designed to evaluate the above analysis. DLL's waveforms around the lock condition are shown in Fig.7. To prove the validation of (19) and (20), simulation has been done for $\mathrm{N}=8,12,16,20$ in DLLs ( $\mathrm{N}$ is number of delay stages in VCDL). The related waveforms have been shown in Fig.8. Both simulation and theoretical predictions show that jitter of DLL due to mis match of delay cells is maximum at middle of VCDL $(X=N / 2)$ and minimu $m$ at start and end of $\operatorname{VCDL}(\mathrm{X}=0$ and $\mathrm{X}=\mathrm{N})$.

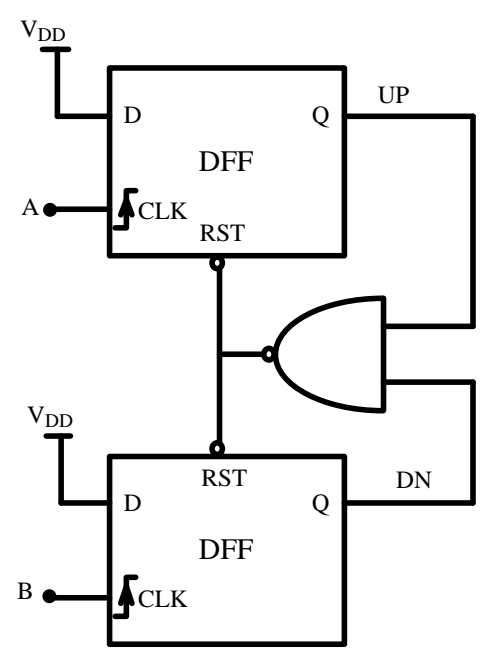

Fig. 5: Conventional PFD which is used in the design of DLL

\section{Conclusions}

In this paper noise and mismatches of conventional delay cells have been examined. First, time domain equations of related to noise and mismatches of conventional delay cells have been expressed. Then, these equations were used to calculate jitter of DLL due to mis match and noise of delay cells. To evaluate these equations a conventional DLL has been designed in TSMC 0.18um CMOS Technology. Simulation results prove the accuracy of theoretical predictions.

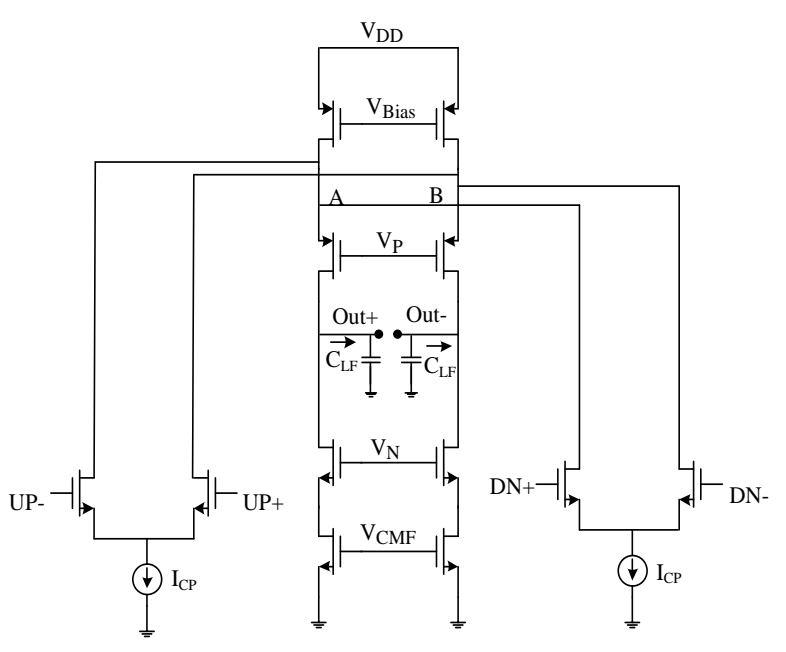

Fig. 6: Charge pump circuit for designing DLL
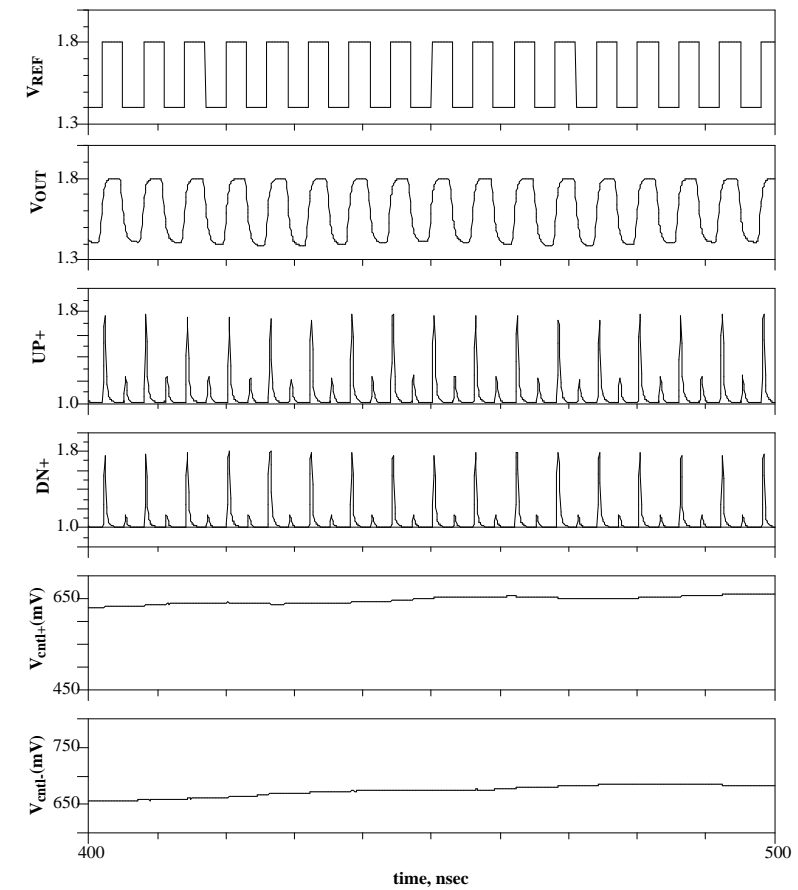

Fig. 7: Waveforms of DLL near lock condition

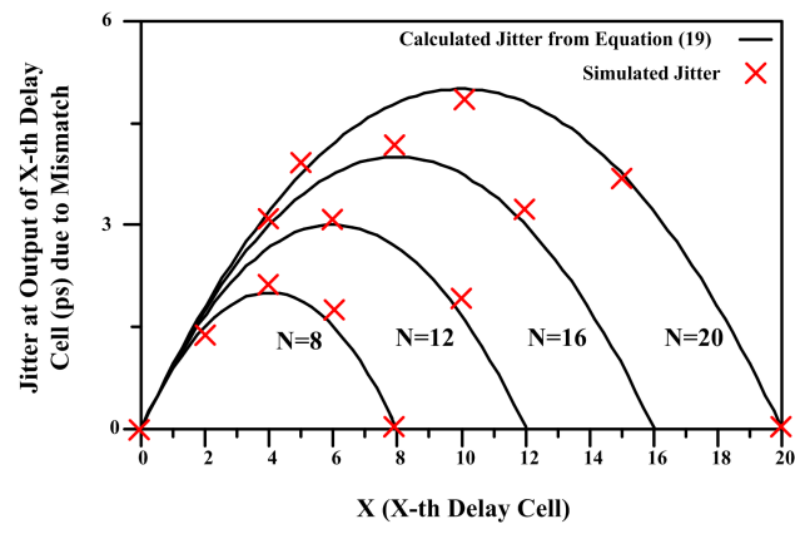

Fig. 8: Jitter at out put of $\mathrm{X}$-th delay cell due to mismatch versus $\mathrm{X}$ for different $\mathrm{N}$ 


\section{Acknowledgments}

The authors would like to thank the anonymous reviewers for their careful reading of this paper and for their helpful comments. This work was supported in part by the Micro-Electronic Groups, Faculty of Electrical Engineering of Babol Noshirvani University of Technology.

\section{References}

[1] M. Gholami, Gh. Ardeshir and H. Ghonoodi, "A novel architecture for low voltage-low power DLLbased frequency multipliers", IEICE Electron. Express, Vol. 8, No. 11, pp.859-865, (2011).

[2] Won-Joo Yun; Hyun-Woo Lee; Dongsuk Shin; Suki Kim; , "A 3.57 Gb/s/pin Low Jitter All-Dig ital DLL With Dual DCC Circuit for GDDR3 DRAM in 54-nm CMOS Technology," Very Large Scale Integration (VLSI) Systems, IEEE Transactions on , vol.19, no.9, pp.1718-1722, Sept. 2011.

[3] J. Lee, K. S. Kundert, and B. Razavi, "Analysis and modeling of bangbang clock and data recovery circuits," IEEE J. Solid-State Circu its, vol. 39, pp. 1571-1580, Sep. 2004.

[4] Oh, K.-I.; Kim, L.-S.; Park, K.-I.; Jun, Y.-H.; Kim, K.; , "Low-jitter multi-phase digital DLL with closest edge selection scheme for DDR memory interface," Electronics Letters , vol.44, no.19, pp.1121-1123, September 112008.

[5] C. Kim, I.-C. Hwang, and S.-M. Kang, "A lowpower s mall-area 7.28-ps-jitter 1-GHz DLL-based clock generator," IEEE J. Solid-State Circuits, vol. 37, pp. 1414-1420, Dec. 2002.

[6] Razavi B., "RF Microelectronics", Second Edition, PRENTICE HALL, 2012.

[7] Gholami, M.; Fallah, M., "Increasing frequency of ring oscillators by using negative capacitors," Electronics Letters , vol.48, no.18, pp.1109,1110, August 30 2012. DOI: 10.1049/el.2012.2261.

[8] Gholami, Mohammad and Rahimpour, Hamid., "Design and analysis of negative capacitor by using MOSFETs," International Journal of Electronics, pp.1,11, 2013. DOI: 10.1080/00207217.2013. 817023.

[9] Rahimpour, Hamid, Mohammad Gholami, Hossein Miar-Naimi, and Gholamreza Ardeshir. "All dig ital fast lock DLL-based frequency multiplier." Analog Integrated Circuits and Signal Processing (2013): 18. DOI: $10.1007 / \mathrm{s} 10470-013-0205-9$.

[10] Gholami, Mohammad, Hamid Rahimpour, Gholamreza Ardeshir, and Hossein Miar - Naimi. "A new fast - lock, low - jitter, and all - digital frequency synthesizer for DVB - T receivers."
International Journal of Circuit Theory and Applications (2013). DOI: 10.1002/cta.1958

[11] Gholami, Mohammad, Hamid Rahimpour, Gholamreza Ardeshir, and Hossein MiarNaimi. "Digital delay locked loop-based frequency synthesiser for Digital Video BroadcastingTerrestrial receivers." Institution of Engineering and Technology (2013). DOI: 10.1049/ietcds.2013.0169

[12] Lee, M.-J.E.; Dally, W.J.; Greer, T.; Hiok-Tiaq Ng; Farjad-rad, R.; Poulton, J.; Senthinathan, R., "Jitter transfer characteristics of delay-locked loops theories and design techniques," Solid-State Circuits, IEEE Journal of , vol.38, no.4, pp.614,621, Apr 2003.

[13] Won-Joo Yun; Hyun-Woo Lee; Shin, Dongsuk; Suki Kim, "A $3.57 \mathrm{~Gb} / \mathrm{s} /$ pin Low Jitter All-Dig ital DLL With Dual DCC Circuit for GDDR3 DRAM in 54-nm CMOS Technology," Very Large Scale Integration (VLSI) Systems, IEEE Transactions on , vol.19, no.9, pp.1718,1722, Sept. 2011.

[14] Wu Gao; Deyuan Gao; Brasse, D.; Hu-Guo, C.; Yann $\mathrm{Hu}$, "Precise Multiphase Clock Generation Using Low-Jitter Delay-Locked Loop Techniques for Positron Emission Tomography Imaging," Nuclear Science, IEEE Transactions on , vol.57, no.3, pp.1063,1070, June 2010

[15] Weigandt, T.C.; Beomsup Kim; Gray, P.R.; , "Analysis of timing jitter in CMOS ring oscillators," Circuits and Systems, 1994. ISCAS '94., 1994 IEEE International Sy mposium on , vol.4, no., pp.27-30 vol.4, 30 May-2 Jun 1994.

[16] Gholami M.; "A Novel Low Power A rchitecture for DLL-Based Frequency Synthesizers," Circuits, Systems, and Signal Processing: Volume 32, Issue 2 (2013), Page 781-801. DOI: 10.1007/s00034-0129488-9.

\section{Authors' Profiles}

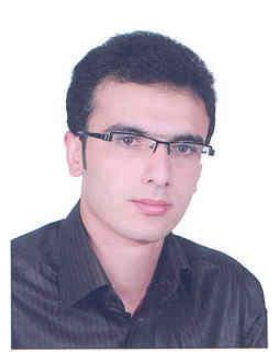

Mohammad Gholami was born in Babol, Iran in 1986. He received the B.Sc. in Electronic Engineering from Mazandaran University (Babol Noshirvani University of Technology), Babol, Iran in 2008. $\mathrm{He}$ also received the M.Sc. in Micro Electronic field from Sharif University of Technology, Tehran, Iran in 2010. Now he is a Ph.D. student in the field of Analog Circuit Design in Babol Noshirvani University of Technology, Babol, Iran. From 2013 he has been a member of the Electrical and Electronic Engineering Faculty at Mazandaran University. His current research interests include the analysis and design of radio frequency CMOS integrated circuits and systems, mixed 
signal circuit design, digital and analog circuit design and designing of DLL or PLL based frequency synthesizers.

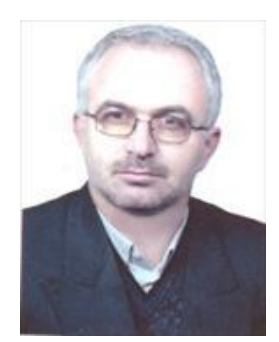

\section{Gholamreza Ardeshir}

He recieved his B.Sc. from Ferdosi University (Mashhad-Iran) in 1989, M.Sc. from Tarbiat Modares University (Tehran-Iran) in 1993 and $\mathrm{Ph} . \mathrm{D}$ from University of surry (Guildford-UK) in 2003 respectively. Since 1994, He has been a member of Electrical Engineering Faculty at Babol Noshirvani University of Technology. His research interests are VLSI circuit design and signal processing.

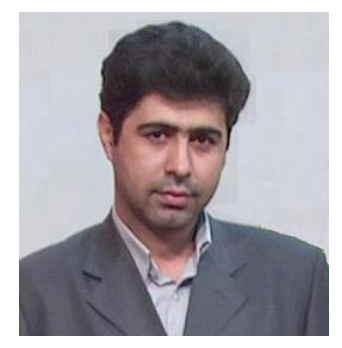

\section{Hossein Miar-Naimi}

He was born in Chalous, Iran, in 1972. He received the B.Sc. degree from Sharif University of Technology, Tehran, Iran, in 1994, the M.Sc. degree from Tarbiat Modares University, Tehran, in 1996 and the Ph.D. degree from Iran University of Science and Technology, Tehran, in 2002, all in electrical engineering. Since 2003 he has been a Member of the Electrical and Electronics Engineering Faculty, Babol University of Technology. His research interests are analog CMOS integrated circuit design, RF and microwave microelectronics.

How to cite this paper: Mohammad Gholami, Gholamreza Ardeshir, Hossein Miar-Naimi,"A Noise and Mismatches of Delay Cells and Their Effects on DLLs", International Journal of Intelligent Systems and Applications(IJISA), vol.6, no.5, pp.37-43, 2014. DOI: 10.5815/ijisa.2014.05.03 